\title{
Performance evaluation of the pixel wing model for the insect wing's camber
}

\author{
Minato Onishi ${ }^{1}$, Daisuke Ishihara ${ }^{2 *}$ \\ ${ }^{1}$ Department of Computer Science and System Engineering, Kyushu Institute of Technology \\ ${ }^{2}$ Department of Intelligent and Control Systems, Kyushu Institute of Technology \\ ${ }^{*}$ ishihara@mse.kyutech.ac.jp
}

Received: February 3, 2021; Accepted: June 14, 2021; Published: July 7, 2021

\begin{abstract}
In insect flapping wings, the camber deformation is caused by the aerodynamic forces. Since the camber will improve the aerodynamic performance of Flapping Wing Nano Air Vehicles (FWNAVs), it is important to elucidate the passive mechanism of the cambering. The pixel wing model consisting of a structured mesh using shell elements that can simulate the camber deformation caused by the fluid-structure interaction has been proposed for the purpose of computational efficiency. In this study, the performance of the pixel wing model is evaluated as the pixel model resolution is changed. The minimum pixel model resolution is determined such that it can keep enough magnitude of camber compared to actual insects. Furthermore, it is found that the cambering of the pixel wing model can be effectively changed using the wing's chord-wise flexural stiffness given by the root vein pixels and the thickness of the wing membrane pixels.
\end{abstract}

Keywords: Insect flapping flight, Pixel wing model, Camber, Shell element, Finite element method

\section{Introduction}

In insect wings, the wing membranes are supported by the complicated network of the veins. Any deformation in the insect wings during their flapping motion will be caused passively by the external mechanical forces such as the inertial and aerodynamic forces, since the wings have no interior muscle [1]. Many literatures have reported the essential importance of the wing's characteristic deformations such as the twisting and the cambering for creating the insect's flight ability [2-5]. The magnitude of the camber is defined as the ratio of the wing's height $C_{\mathrm{h}}$ to the wing's chord length $C_{\mathrm{l}}$ as shown in Fig. 1, where $C_{\mathrm{h}}$ is the distance between the points $\mathrm{C}$ and $\mathrm{H}, \mathrm{C}_{\mathrm{l}}$ is the distance between the points $\mathrm{A}$ and $\mathrm{B}$, and the sign of the camber is positive if the shape of the camber is concave along the direction of the flapping translation [6]. It is important to elucidate the passive mechanism of the cambering for the performance improvement of Flapping Wing Nano Air Vehicles (FWNAVs) mimicking the insect flapping flight, since the camber can increase the aerodynamic performance $[4,5]$. 


\section{Journal of Advanced Simulation in Science and Engineering}

Some insect wing models consisting of beam and shell elements have been proposed [7, 8]. In these models, the complicated network of the veins is represented using the beam elements, and the wing membrane is represented using the unstructured elements. However, the usage of the unstructured elements might impose a sophisticated mesh-moving technique on controlling the fluid mesh surrounding the wing model [9] in the finite element analysis of the fluid-structure interaction. Hence, for the purpose of computational efficiency, the pixel wing model consisting of a structured mesh using shell elements has been proposed [10]. As far as we know, except our previous studies [7, 10], there are few studies on insect wing models that can deform elastically such that they produce sufficient camber compared to actual insects. In this model, the model resolution will have strongly affected on the analysis result because this model is composed of structured mesh similar to the voxel model [11]. Therefore, it is important to evaluated the relationship between the model resolution and the analysis result.

In this study, the performance of the pixel wing model is evaluated by changing the number of pixels, since the deformation of the pixel wing model is sensitive to this number. In this evaluation, the relationship between the pixel model resolution and the magnitude of the camber is investigated, and the minimum pixel model resolution is determined such that it can keep enough camber compared to actual insects. Furthermore, it is found that the cambering of the pixel wing model can be effectively changed using the wing's chord-wise flexural stiffness determined by the root vein pixels and the thickness of the wing membrane pixels.

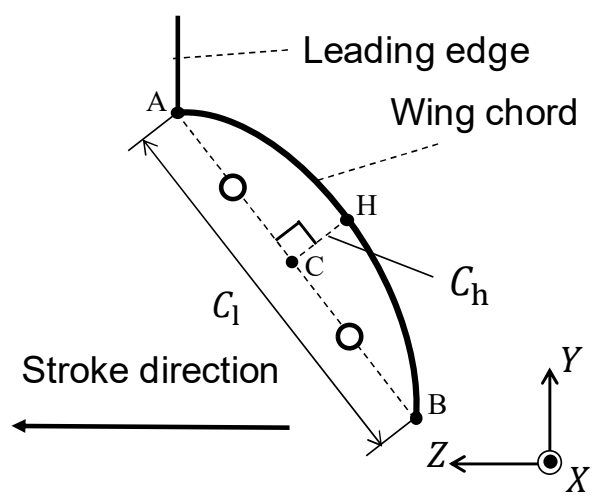

Figure 1: Schematic of the camber deformation.

\section{Definition of the pixel wing model}

The pixel wing model consists of a structured mesh using shell elements as shown in Fig. 2. Each element represents a pixel. The wing span length is $R_{\mathrm{w}}$, and the wing chord length is $c_{\mathrm{w}}$. The wing base is the area at $x=0$ along the $y$-axis, where the fixed support is imposed. The complicated network of veins is simplified by the leading edge, the center vein, and the root vein based on Ref. [7] that represent the functionally distinct domains of insect wings as the supporting structures. Since the veins occupy the very narrow wing's area compared to the wing membrane in actual insects $[12,13]$, the area of pixels describing these domains is minimized as shown in Fig. 3. The rest of the pixels represents the wing membrane. The different set of the pseudo-elastic material properties is given to the pixels in each domain as shown in Fig. 3 for the purpose of giving the different mechanical behaviors among the veins (the leading edge, the center vein, and the root vein) and the wing membrane. The Young's modulus of the 


\section{Journal of Advanced Simulation in Science and Engineering}

leading edge is determined such that the flexural rigidity along the wing's span direction is equivalent to that of an actual insect [14]. The Young's modulus of the root vein is determined such that the flexural rigidity along the wing's chord direction is equivalent to that of an actual insect [14]. The Young's modulus of the center vein is determined such that its flexural rigidity is equal to that of a most significant vein in the center domain of an actual insect's wing [12]. The Young's modulus of the wing membrane is determined from the material property of an actual insect [15]. The nondimensional parameter $r_{\mathrm{v}}$ describes the pixel model resolution, which is defined as the ratio of the area of three veins $S_{\mathrm{v}}$ and the area of the wing $S_{\mathrm{w}}$ as follows:

$$
r_{\mathrm{v}}=\frac{S_{\mathrm{v}}}{S_{\mathrm{w}}}
$$

In this model, a size of each pixel is determined by $r_{\mathrm{v}}$, and it increases as $r_{\mathrm{v}}$ increases. Therefore, the width of veins increases as $r_{\mathrm{v}}$ increases.

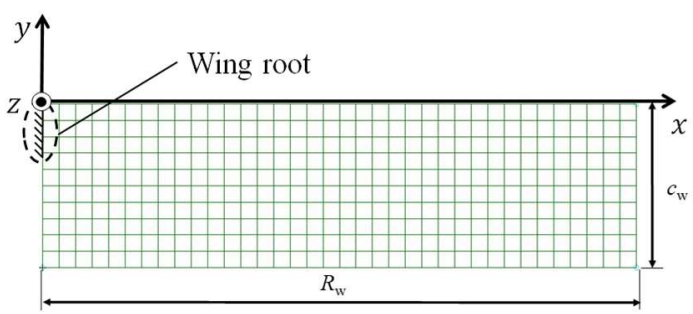

Figure 2: Pixel wing model consisting of a structured mesh using shell elements. Each element represents a pixel. The wing base is defined as the area, where the fixed support is imposed.

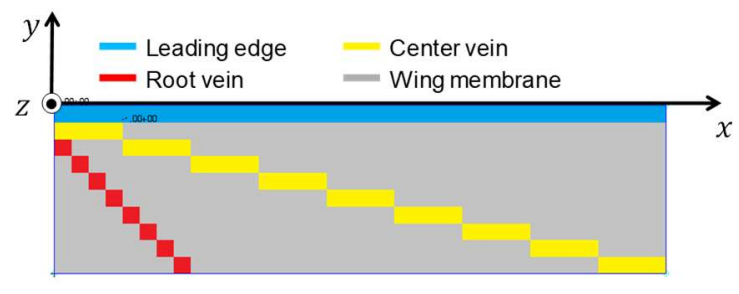

Figure 3: Pseudo-elastic material properties in the pixel wing model. The colors represent the different functional roles of the pixels, and each of them corresponds to the different set of the material properties.

\section{Quasi static modeling of aerodynamic force}

A flapping wing model is schematically shown in Fig. 4. As shown in this figure, the flapping axis coincides with the $y$-axis, and the leading edge of the wing model flaps and translates in the stroke plane with a stroke angle $\Phi$. The magnitude of the twisting or the feathering angle $\theta$ is defined as the angle between the current wing's chord and the initial wing's chord. The flapping angular displacement $\varphi$ is defined as the angle between the leading edge and the $x$-axis. The time history of the flapping angular velocity $\mathrm{d} \varphi / \mathrm{d} t$ can be expressed using the trapezoidal function as shown in Fig. 5 [16], which is based on the actual observation [17]. In this time history, the dynamic pressure $P$ is dominant at the middle of each half-stroke, since the wing flaps with a constant and maximum speed $\omega_{\max }$ during this period. Therefore, in this study, the dynamic pressure $P$ can be given using a quasi-static approximation as

$$
P=\frac{1}{2} C_{\mathrm{D}} \rho^{\mathrm{f}} V_{\max }^{2}
$$




$$
V_{\max }=\omega_{\max } r=\frac{8 \Phi r}{3 T_{\varphi}}
$$

where $C_{\mathrm{D}}$ is the drag coefficient for the flat plate, $\rho^{\mathrm{f}}$ is the fluid mass density, $r$ is the distance from the wing base, $\Phi$ is the stroke angle, and $T_{\varphi}$ is the flapping period. The acceleration and deceleration time $t_{\varphi}$ are set to $T_{\varphi} / 8$, which is a typical value [16]. $V_{\max }$ is the maximum flapping speed at the point $r$ corresponding to $\omega_{\max }$. Since this dynamic pressure causes the large deformation in the wing model, a finite element method considering the geometrical nonlinearity is used.

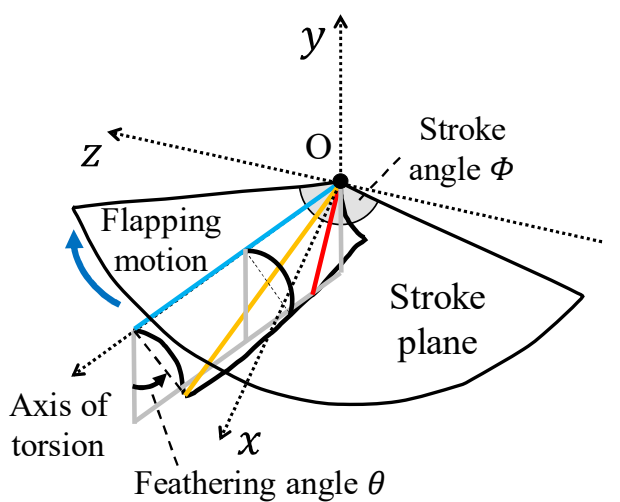

Figure 4: Schematic of the flapping motion in the wing.

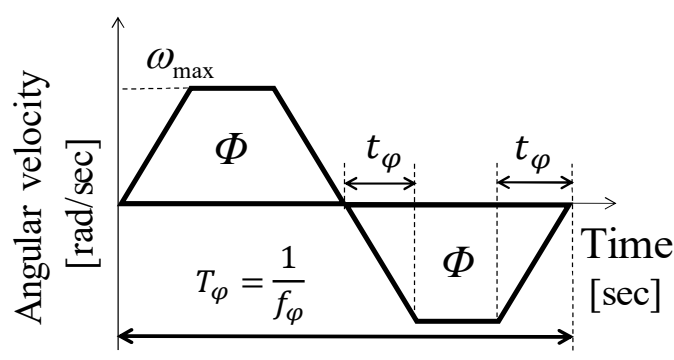

Figure 5: Time history of the flapping angular velocity.

\section{Analysis of the pixel wing model}

\subsection{Effect of the pixel model resolution}

The wing's geometric parameters, the material properties of the wing's membrane, and the kinematic parameters of the wing's flapping are given based on the data obtained from the actual insects $[12,13,15,17,18]$. The Young's modulus of the vein and the leading edge are given using the flexural rigidities of an actual insect wing [14] following the procedure described in Section 2. The leading edge is determined using the flexural rigidity along the wing's span-wise direction $G_{\mathrm{s}}$. The root vein is determined using the flexural rigidity along the wing's chord-wise direction $G_{\mathrm{c}}$. The center vein is determined using the flexural rigidity of the most significant center vein $[12,15]$. The parameters used in this study are summarized in Table 1.

Figures 6 and 7 show the deformation of the wing model with the pixel model resolutions $r_{\mathrm{v}}=8 \%$ and $r_{\mathrm{v}}=22 \%$, respectively. Figure 8 shows the $z$-displacement of the wing chord at the normalized position $r^{*}=r / R_{\mathrm{W}}=80 \%$, where $r$ is the distance from the wing base, and $R_{\mathrm{w}}$ is the wing's span length. As shown in these figures, the wing deforms greater to form more significant camber as $r_{\mathrm{v}}$ decreases. Figure 9 shows the distribution of the camber along the wing's span-wise direction for the various $r_{\mathrm{v}}$. As shown in this figure, the magnitude of the camber increases throughout the wing as $r_{\mathrm{v}}$ decreases. Therefore, the ability of the pixel wing model for simulating the camber deformation depends on the pixel model resolution. This dependency can be explained as follows. 
Table 1: Parameters of the pixel wing model

\begin{tabular}{|l|c|}
\hline Wing span length $R_{\mathrm{w}}[\mathrm{m}]$ & 0.0113 \\
\hline Wing chord length $c_{\mathrm{w}}[\mathrm{m}]$ & 0.00311 \\
\hline Thickness of wing membrane $t_{\mathrm{m}}[\mu \mathrm{m}]$ & 1.40 \\
\hline Young's modulus of wing membrane $[\mathrm{GPa}]$ & 1.0 \\
\hline Poisson's ratio $v$ & 0.49 \\
\hline Stroke angle $\Phi[\mathrm{deg}]$ & 108 \\
\hline Flapping frequency $f_{\varphi}\left(=T_{\varphi}:\right.$ flapping period) $[\mathrm{Hz}]$ & 161 \\
\hline Fluid mass density $\rho^{\mathrm{f}}\left[\mathrm{kg} / \mathrm{m}^{3}\right]$ & 1.205 \\
\hline Drag coefficient $C_{\mathrm{D}}$ & 1.19 \\
\hline Flexural rigidity of along the wing's span-wise direction $G_{\mathrm{s}}\left[\mu \mathrm{Nm}^{2}\right]$ & 4.0 \\
\hline Flexural rigidity of along the wing's chord-wise direction $G_{\mathrm{c}}\left[\mu \mathrm{Nm}^{2}\right]$ & 0.35 \\
\hline Flexural rigidity of the most significant center vein $\left[\mathrm{nNm}^{2}\right]$ & 0.26 \\
\hline
\end{tabular}

Let us consider the rotation of the root vein around its longitudinal axis. As shown in Fig. 10 , this can be evaluated using the rotational degrees of freedoms $\theta_{y}$ of the nodes located on the longitudinal axis of the root vein. Figure 11 shows the relationship between $\theta_{y}$ and the distance from the base of this vein. As shown in this figure, $\theta_{y}$ monotonically increases as $r_{\mathrm{v}}$ decreases. This is because the mean of the width of the root vein decreases as $r_{\mathrm{v}}$ decreases, meaning that the torsional stiffness around the longitudinal axis of the root vein decreases. As discussed in our previous study [10], the rotation of the root vein can increase the camber deformation.

The computing times for analyzing the pixel models with various $r_{\mathrm{v}}$ are summarized in Table 2. As shown in this table, the computing time increases as $r_{\mathrm{v}}$ decreases because of increase of the number of degrees of freedoms to be analyzed. As shown in Fig. 9, the present camber distributions given by the pixel wing model with $r_{\mathrm{v}}$ equal to or less than $12 \%$ are comparable to those observed in the actual insects [6]. Therefore, $r_{\mathrm{v}}=12 \%$ is determined as the minimum pixel model resolution. The computing time for this model can be reduced by $23 \%$ approximately that for the pixel model with the lowest $r_{\mathrm{v}}=8 \%$.

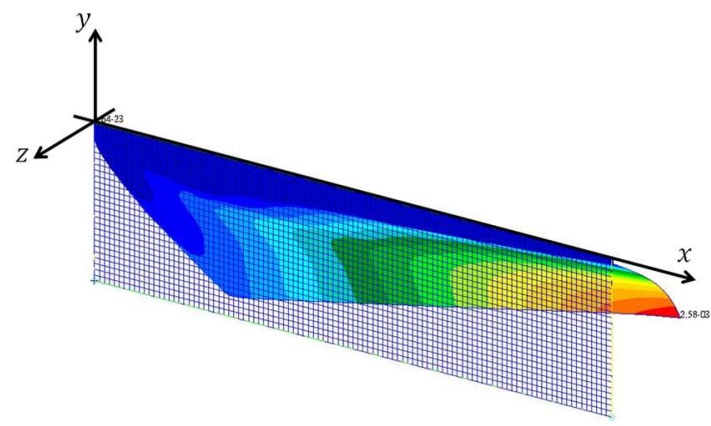

Figure 6: Deformation of wing model with $r_{\mathrm{v}}$ $=8 \%$.

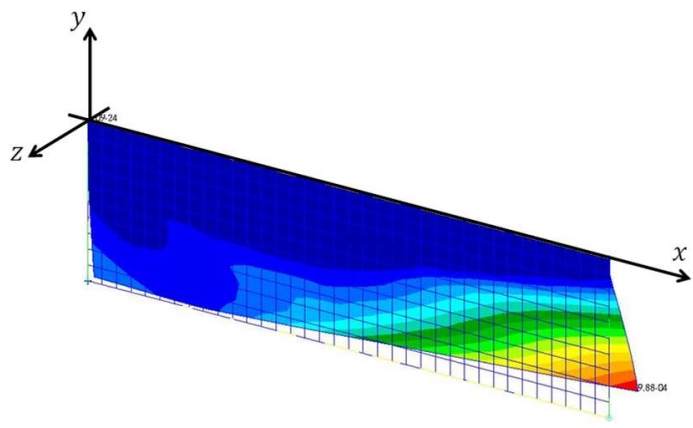

Figure 7: Deformation of wing model with $r_{\mathrm{v}}$ $=22 \%$ 


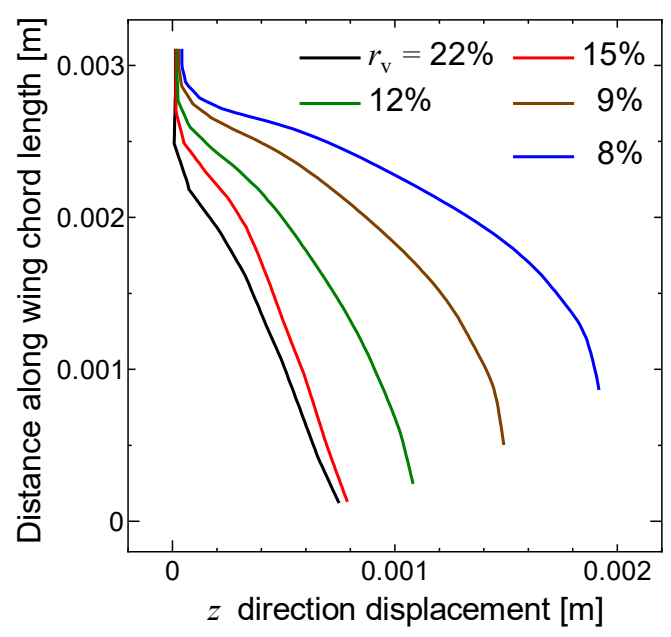

Figure 8: Displacements in the $z$-direction of the wing chord at the position of $80 \%$ from the wing base.

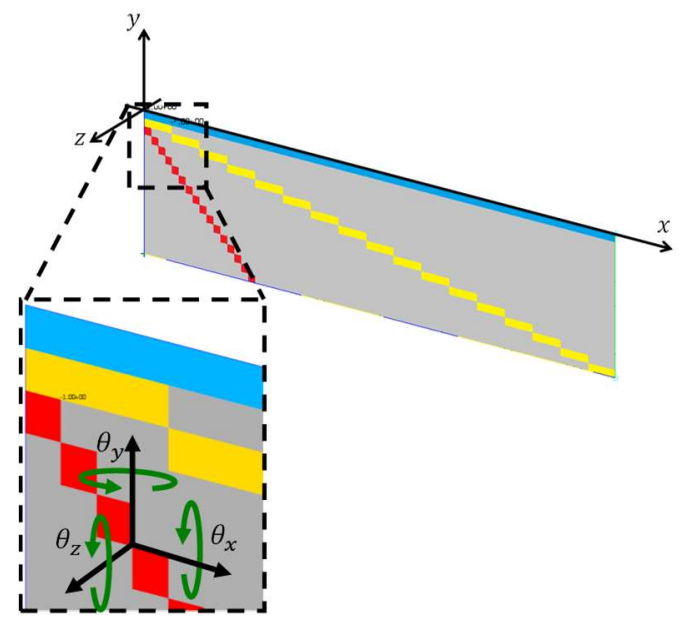

Figure 10: The rotational displacements of the nodes located along the central axis of the root vein .

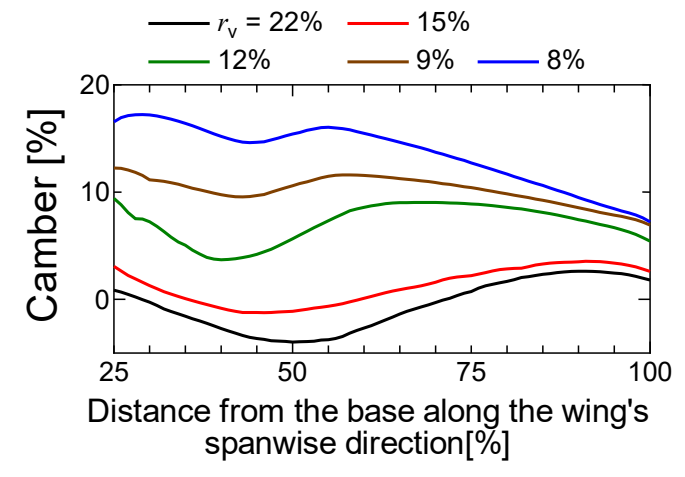

Figure 9: Camber distribution against distance from the base along the wing's spanwise direction.

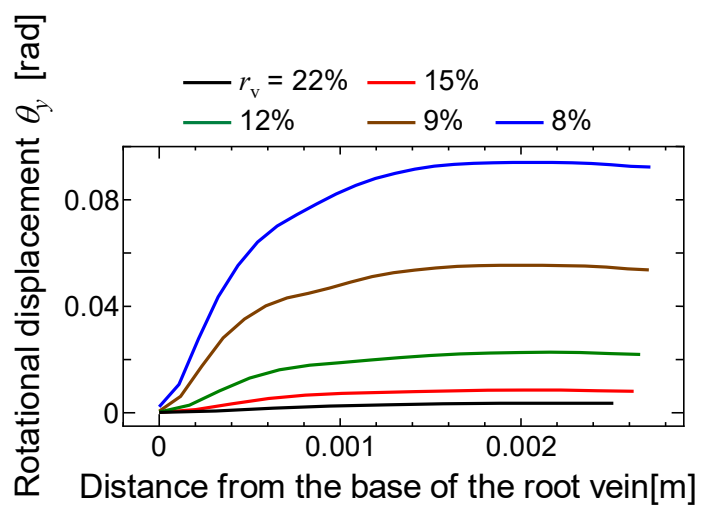

Table 2: Computing times for analyzing the pixel model with various resolutions

\begin{tabular}{|c|c|}
\hline Pixel model resolution $r_{\mathrm{v}}[\%]$ & Computing time (wall time) [sec] \\
\hline 22 & 486.16 \\
\hline 15 & 663.23 \\
\hline 12 & 965.59 \\
\hline 9 & 1167.26 \\
\hline 8 & 1250.68 \\
\hline
\end{tabular}




\section{Journal of Advanced Simulation in Science and Engineering}

\subsection{Effect of the flexural rigidity along the wing's chord-wise direction and the thickness of the wing membrane}

There exist wide variations in the wing's parameters such as the flexural rigidity along the wing's chord-wise direction and the thickness of the wing membrane obtained from actual insects. Therefore, the purpose of this section is to improve the simulation ability of the pixel wing model by changing these parameters within the range of actual insects. Here, the pixel wing model in the case of $r_{\mathrm{v}}=12 \%$ is used.

The flexural rigidity along the wing's chord-wise direction $G_{\mathrm{c}}$ is varied from $0.20 \mu \mathrm{Nm}^{2}$ to $0.50 \mu \mathrm{Nm}^{2}$. As described in Section 2, the Young's modulus is used to change $G_{\mathrm{c}}$ of the root vein. Figure 12 shows the relationship between $G_{\mathrm{c}}$ and the average camber. Figures 13 and 14 show the deformations of the wing model with two extremes of $G_{\mathrm{c}}=0.20 \mu \mathrm{Nm}^{2}$ and $0.50 \mu \mathrm{Nm}^{2}$, respectively. As shown in these results, the camber increases as $G_{\mathrm{c}}$ decreases because of the same mechanism of the increase of the camber as the decrease of $r_{\mathrm{v}}$. That is, it is because the torsional stiffness of the root vein decreases as $G_{\mathrm{c}}$ decreases.

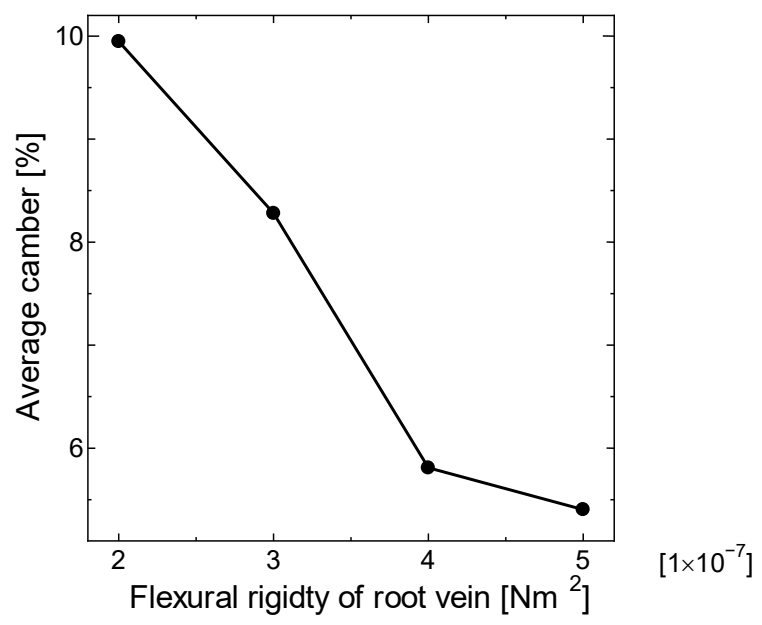

Figure 12: The relationship between the average camber and the flexural rigidity along the wing's chord-wise direction.

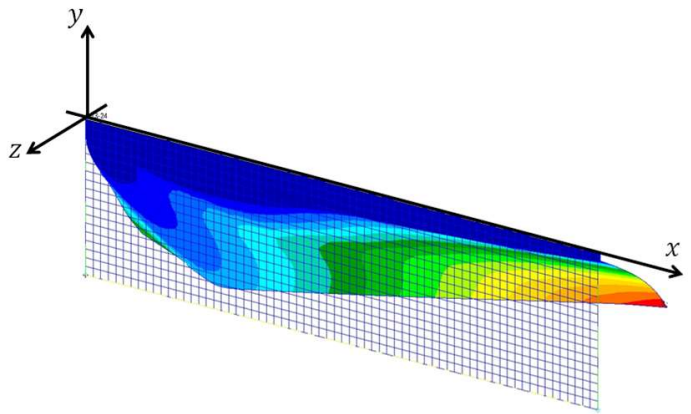

Figure 13: Deformation of wing model with $G_{\mathrm{c}}=0.20 \mu \mathrm{Nm}^{2}$.

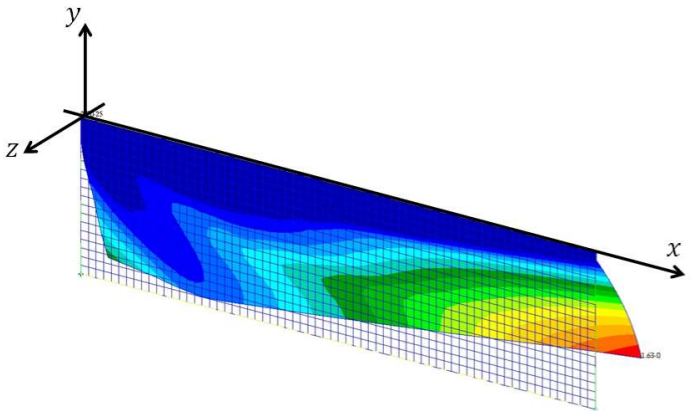

Figure 14: Deformation of wing model with $G_{\mathrm{c}}=0.50 \mu \mathrm{Nm}^{2}$. 
The thickness of the wing membrane $t_{\mathrm{m}}$ is varied from $1.25 \mu \mathrm{m}$ to $5.00 \mu \mathrm{m}$. Figure 15 shows the relationship between $t_{\mathrm{m}}$ and the average camber. Figures 16 and 17 show the deformation of the wing model with two extremes of $t_{\mathrm{m}}=1.25 \mu \mathrm{m}$ and $5.00 \mu \mathrm{m}$, respectively. As shown in these results, the average camber decreases as $t_{\mathrm{m}}$ increases. This is because the difference between the veins and the membrane decreases as $t_{\mathrm{m}}$ increases, then the wing's structure approaches the uniform plate.

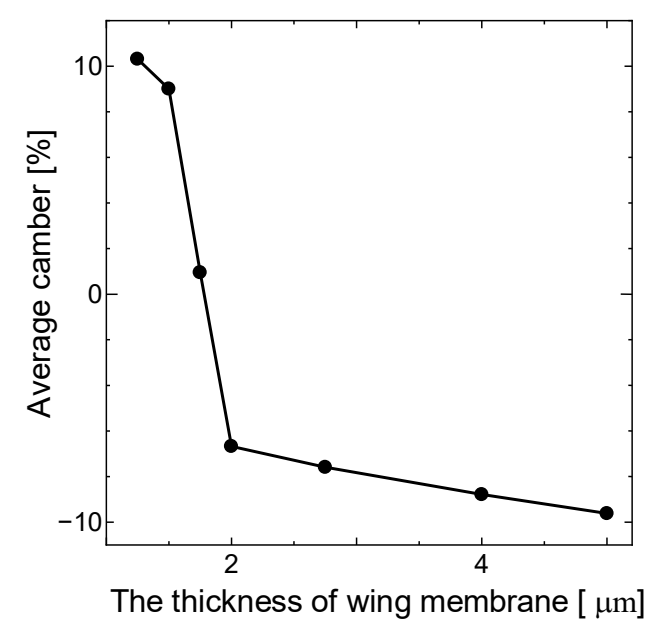

Figure 15: The relationship between the average camber and the thickness of wing membrane.

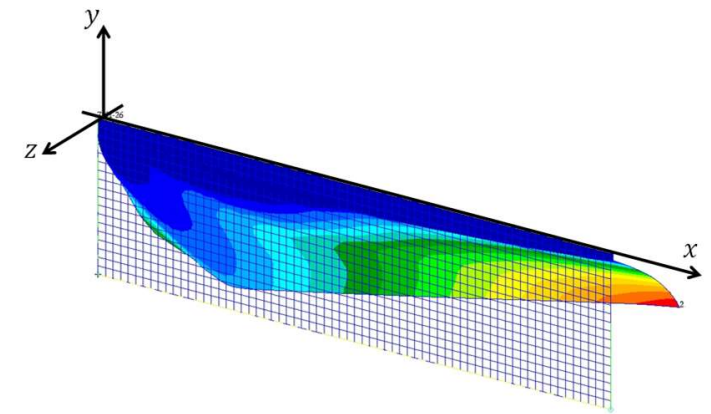

Figure 16: Deformation of wing model with $t_{\mathrm{m}}=1.25 \mu \mathrm{Nm}^{2}$.

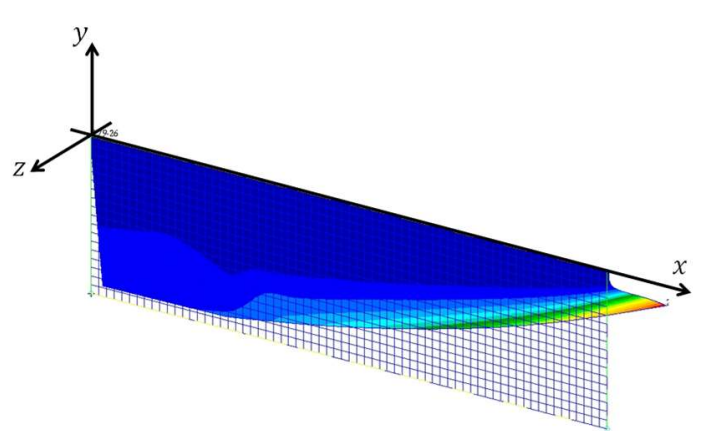

Figure 17: Deformation of wing model with $t_{\mathrm{m}}=5.00 \mu \mathrm{Nm}^{2}$.

\section{Concluding remarks}

In this study, the performance of the pixel wing model was evaluated using the finite element method considering the geometrical nonlinearity. The relationship between the camber deformation and the pixel model resolution has shown that the camber generated by this model strongly depends on the pixel model resolution. This is because the torsional flexibility of the 


\section{Journal of Advanced Simulation in Science and Engineering}

root vein increases as the pixel model resolution decreases. The minimum pixel model resolution was determined using these results such that it can produce enough camber deformation compared to actual insects. The other important two parameters of the pixel wing model were found to control the camber. One is the flexural rigidity along the wing's chord-wise direction, and the other is the thickness of the wing membrane. The magnitude of the camber can be improved by decreasing these two parameters. In our future work, the pixel wing model will be used in the finite element analysis of strong coupling between the wing and the surrounding fluid to elucidate the passive cambering mechanism.

\section{Acknowledgement}

This work was supported by JSPS KAKENHI Grant Number $20 \mathrm{H} 04199$.

\section{References}

[1] R. J. Wooton, R. C. Herbert, P. G. Young, K. E. Evans: Approaches to the structural modeling of insect wings. Philosophical Transactions of the Royal Society of London B, 358, pp. 1577-1587.

[2] J. Young, S. M. Walker, R. J. Bomphrey, G. K. Taylor, A. L. R. Thomas: Details of insect wing design and deformation enhance aerodynamic function and flight efficiency, Science, 325, pp.1549-1552, 2009.

[3] L. Zheng, T. L. Hedrick, R. Mittal, Time-Varying Wing-Twist Improves Aerodynamic Efficiency of Forward Flight in Butterflies, PLoS ONE, 8, 1, e53060, 2013.

[4] T. Nakata, R. Noda, H. Liu: Effect of twist, camber and spanwise bending on the aerodynamic performance of flapping wings, Journal of Biomechanical Science and Engineering, 13, 2, 2018.

[5] G. Du, M. Sun M: Effects of the wing deformation on the aerodynamic forces in hovering hoverflies, The Journal of Experimental Biology, 213, pp. 2273-2283, 2010.

[6] S. M. Walker, A. L. R. Thomas, G. K. Taylor: Deformable wing kinematics in free-flying hoverfly, Journal of the Royal Society Interface, 7, pp. 131-142, 2010.

[7] D. Ishihara, J. Yokota, M. Onishi, T. Niho, T. Horie: A shape simplification modeling of the cambering in insect's flapping wings using beam and shell, Transactions of the Japan Society for Computational Engineering and Science, 2018, pp. 20180018, 2018.

[8] A. Shahzad, F-B. Tian, J. Young, J. C. S. Lai: Effect of hawkmoth-like flexibility on the aerodynamic performance of flapping on the performance of flapping wings with difference shapes and aspect ratios. Physics of Fluids, 30, 091902, 2018.

[9] D. Ishihara, A. Goto, M. Onishi, T. Horie, T. Niho: Element-quality-based stiffening for the pseudoelastic mesh-moving technique, International Journal of Computational Methods, 17, 14, 1850146, 2020.

[10] M. Onishi, D. Ishihara: Modeling the cambering of the flapping wings of an insect using rectangular shell finite elements, Journal of Advance Simulation in Science and Engineering, 7, 1, pp. 181-188, 2020.

[11] T. W. Dawson, K. Caputa, M. A. Stuchly: Influence of Human Model Resolution on 
Computed Currents Induced in Organs by $60-\mathrm{Hz}$ Magnetic Fields, Bioelectromagnetics, 18, pp. 478-490, 1997.

[12] H. Tanaka, J. P. Whitney, R. J. Wood: Effect of flexural and torsion wing flexibility on lift generation in hoverfly flight. Integrative and Comparative Biology, 51, pp. 142-150, 2011.

[13] H. Tanaka, R. J. Wood: Fabrication of corrugated artificial insect wings using laser micromachined molds, Journal of Micromechanics and Microengineering, 20, 075008, 2010.

[14] S. A. Combes, T. L. Daniel: Flexural stiffness in insect wings I. Scaling and the influence of the wing venation. The Journal of Experimental Biology, 206, pp. 2979-2987, 2003.

[15] S. A. Wainwright, W. D. Biggs, J. D. Currey, J. M. Gosline: Mechanical Design in Organism, Princeton University Press, 1982.

[16] D. Ishihara, T. Horie, T. Niho: An experimental and there-dimensional computational study on the aerodynamic contribution to the passive pitching motion of flapping wings in hovering flies, Bioinspiration \& Biomimetics, 9, 046009, 2014.

[17] C. P. Ellington: The aerodynamics of hovering insect flight III. Kinematics, Philosophical Transactions of the Royal Society of London B, 305, 1122, pp. 41-78, 1984.

[18] A. R. Ennos: The kinematics and aerodynamics of the free flight of some Diptera. The Journal of Experimental Biology, 142, pp. 49-85, 1989. 